\title{
Correction to: Soil Classification and Distribution
}

Hitoshi Shinjo and Yusuke Takata

\section{Correction to:}

\section{Chapter 3 in: R. Hatano et al. (eds.), The Soils of Japan, World Soils Book Series,} https://doi.org/10.1007/978-981-15-8229-5_3

In the original version of the book, text that was inadvertently published in Table 3.1 (Chapter 3) has been deleted. The correction chapter and the book have been updated.

Table 3.1 Corrleation of soil classification system of Japan (2017) with WRB (2015) and Soil Taxonomy (2014)

\begin{tabular}{|c|c|c|c|c|}
\hline \multicolumn{3}{|c|}{ Soil classification system of Japan (2017) } & \multirow{4}{*}{$\begin{array}{l}\text { World reference base for soil } \\
\text { resources (2015) }\end{array}$} & \multirow[t]{4}{*}{ UUSDA Soil Taxonomy (2014) } \\
\hline \multicolumn{3}{|l|}{ Great group } & & \\
\hline & Group & & & \\
\hline & & Subgroup & & \\
\hline \multicolumn{3}{|c|}{ A.【Human-made soils】 } & Technosols, Regosols & (Entisols) \\
\hline & Artifactural soils & & Technosols & (Udorthents) \\
\hline & & Organic & Gabric Technosols & \\
\hline & & Ekrantic & $\begin{array}{l}\text { Linic Technosols } \\
\text { Ekranic Technosols }\end{array}$ & \\
\hline & & Mineral & $\begin{array}{l}\text { Urbic Technosols } \\
\text { Spolic Technosols }\end{array}$ & \\
\hline & Reformed soils & & Regosols (Transportic) & (Udorthents) \\
\hline & & Upland & Regosols (Transportic) & \\
\hline & & Lowland & Regosols (Transportic) & \\
\hline \multicolumn{2}{|l|}{ B. 【Organic soils】 } & & Histosols & Histosols \\
\hline & Peat soils & & Histosols & Histosols \\
\hline & & Sapric & Saplic Histosols & Haplosaprists \\
\hline & & High-moor & $\begin{array}{l}\text { Fibric Histosols } \\
\text { Hemic Histosols }\end{array}$ & $\begin{array}{l}\text { Shagnofibrists } \\
\text { Haplofibrists } \\
\text { Haplohemists }\end{array}$ \\
\hline & & Transitional-moor & $\begin{array}{l}\text { Fibric Histosols } \\
\text { Hemic Histosols }\end{array}$ & $\begin{array}{l}\text { Haplofibrists } \\
\text { Haplohemists }\end{array}$ \\
\hline
\end{tabular}


Table 3.1 (continued)

\begin{tabular}{|c|c|c|c|c|}
\hline \multicolumn{3}{|c|}{ Soil classification system of Japan (2017) } & \multirow{4}{*}{$\begin{array}{l}\text { World reference base for soil } \\
\text { resources (2015) }\end{array}$} & \multirow[t]{4}{*}{ UUSDA Soil Taxonomy (2014) } \\
\hline \multicolumn{3}{|l|}{ Great group } & & \\
\hline & Group & & & \\
\hline & & Subgroup & & \\
\hline & & Low-moor & $\begin{array}{l}\text { Fibric Histosols } \\
\text { Hemic Histosols }\end{array}$ & $\begin{array}{l}\text { Haplofibrists } \\
\text { Haplohemists }\end{array}$ \\
\hline \multirow[t]{20}{*}{ C.【Andosols】 } & & & Andosols, Podzols & Andisols, Spodosols \\
\hline & $\begin{array}{l}\text { Podzolic } \\
\text { Andosols }\end{array}$ & & Andic Podzols & $\begin{array}{l}\text { Aquods, Humods, Orthods, Aquands, } \\
\text { Vitrands, Udands }\end{array}$ \\
\hline & & Epi-peaty & Andic Histic Podzols & Histic Endoaquods, Histic Endoaquands \\
\hline & & Epi-pseudogleyic & Andic Stagnic Podzols & $\begin{array}{l}\text { Lithic Epiauods, Andic Epiaquods, Typic } \\
\text { Epiaquods, Vitraquands, Melanaqunds, } \\
\text { Epiaquands }\end{array}$ \\
\hline & & Aquic & Andic Gleyic Podzols & $\begin{array}{l}\text { Lithic Epiauods, Andic Epiaquods, Typic } \\
\text { Epiaquods, Aquic Haplorthods, } \\
\text { Vitraquands, Melanaqunds, Epiaquands, } \\
\text { Aquic subgroups in Andiosols }\end{array}$ \\
\hline & & Haplic & Andic Podzols & $\begin{array}{l}\text { Andic Haplohumods, Andic Haplorthods, } \\
\text { Andisols except Aquands and Aquic } \\
\text { subgroups }\end{array}$ \\
\hline & $\begin{array}{l}\text { Regosolic } \\
\text { Andosols }\end{array}$ & & Vitric Andosols & Vitrands, Aquands \\
\hline & & Aquic & Gleyic Vitric Andosols & Vitraquands \\
\hline & & Humic & $\begin{array}{l}\text { Melanic Vitric Andosols } \\
\text { Umbric Vitric Andosols }\end{array}$ & Humic Udivitrands \\
\hline & & Thapto-humic & Vitric Andosols & Thaptic Udivitrands \\
\hline & & Haplic & Vitric Andosols & Typic Udivitrands \\
\hline & Gleyed Andosols & & Gleysols (Andic) & Aquands \\
\hline & & Peaty & Histic Gleysols (Andic) & Endoaquands \\
\hline & & Cumulic & Dystric Gleysols (Andic) & $\begin{array}{l}\text { Melanaquands } \\
\text { Endoaquands }\end{array}$ \\
\hline & & Haplic & Dystric Gleysols (Andic) & Typic Endoaquands \\
\hline & Wet Andosols & & $\begin{array}{l}\text { Gleyic Silandic Andosols } \\
\text { Gleyic Aluandic Andosols }\end{array}$ & Aquands \\
\hline & & Peaty & $\begin{array}{l}\text { Histic Gleyic Silandic Andosols } \\
\text { Histic Gleyic Aluandic Andosols } \\
\text { Thapto-histic Gleyic Silandic } \\
\text { Andosols } \\
\text { Thapto-histic Gleyic Aluandic } \\
\text { Andosols }\end{array}$ & Histic Endoaquands \\
\hline & & Thapto-upland & $\begin{array}{l}\text { Gleyic Silandic Andosols } \\
\text { Gleyic Aluandic Andosols }\end{array}$ & Typic Endoaquands \\
\hline & & Endofluvic & $\begin{array}{l}\text { Gleyic Silandic Andosols } \\
\text { Gleyic Aluandic Andosols }\end{array}$ & Typic Endoaquands \\
\hline & & Cumulic & $\begin{array}{l}\text { Histic Gleyic Silandic Andosols } \\
\text { Histic Gleyic Histic Aluandic } \\
\text { Andosols } \\
\text { Gleyic Silandic Andosols } \\
\text { (Melanic) } \\
\text { Gleyic Aluandic Andosols } \\
\text { (Melanic) }\end{array}$ & $\begin{array}{l}\text { Melanaquands } \\
\text { Endoaquands }\end{array}$ \\
\hline
\end{tabular}


Table 3.1 (continued)

Soil classification system of Japan (2017)

Great group

\begin{tabular}{l|l|l|}
\hline & Group & Subgroup \\
\hline & & Haplic \\
\hline & $\begin{array}{l}\text { Non-allophanic } \\
\text { Andosols }\end{array}$ & Anthraquic \\
\hline & & Cumulic \\
\hline
\end{tabular}

\begin{tabular}{l|l|l}
\hline & Thapto-humic & Aluandic Andosols \\
\hline & Humic & Aluandic Andosols \\
\hline & Brown-humic & Aluandic Andosols (Fulvic) \\
& & \\
\hline
\end{tabular}

\begin{tabular}{l|l|l|}
\hline & Aquic \\
\hline & Haplic \\
\hline
\end{tabular}

\begin{tabular}{l|l|l|}
\hline & Allophanic & \\
Andosols & \\
\hline
\end{tabular}

\begin{tabular}{l|l|l|}
\hline & Anthraquic \\
\hline & Thapto-upland \\
\hline
\end{tabular}

\begin{tabular}{l|l|l|}
\hline & Endofluvic \\
\hline & Cumulic \\
\hline & Cumulic \\
\hline & Humic \\
\hline
\end{tabular}

\begin{tabular}{l|l}
\hline \hline & \\
\hline & \\
\hline & \\
\hline & \\
\hline D. & \\
\hline Podzols] & \\
\hline
\end{tabular}

D. 【Podzols】

\begin{tabular}{l|l|l}
\hline D.【Podzols】 & \\
\hline & & Epdzols \\
\hline & & Aquic \\
\hline & & Epi-pseudy \\
\hline & & Pseudogleyic \\
\hline
\end{tabular}

World reference base for soil resources (2015)

Gleyic Silandic Andosols

Gleyic Aluandic Andosols

Aluandic Andosols

Aluandic Andosols

Gleyic Aluandic Andosols

Aluandic Andosols

Silandic Andosols

Silandic Andosols

Silandic Andosols

Silandic Andosols

Siluandic Andosols

Silandic Andosols

Silandic Andosols

Brown-humic

Aquic

Haplic

Pseudogleyic

Histic Podzols
Siluandic Andosols (Melanic)
Silandic Andosols (Fulvic)

Gleyic Silandic Andosols

Silandic Andosols

Podzols

Podzols

Gleyic Podzols

Stagnic Podzols

Stagnic Podzols
UUSDA Soil Taxonomy (2014)

Typic Endoaquands

Alic Hapludands

Melanudands

Anthraquic Melanudands Anthraquic Hapludands

Pachic Melanudands Alic Hapludands

Alic Hapludands

Alic Hapludands

Pachic Fulvudands

Thaptic Fulvudands

Typic Fulvudands

Hydric Fulvudands

Aquic Hapludands

Oxyaquic Hapludands

Alic Hapludands

Udands

Anthraquic Melanudands

Anthraquic Hapludands

Typic Hapludands

Ultic Hapludands

Typic Hapludands

Pachic Melanudands

Typic Hapludands

Thaptic Hapluands

Typic Hapludands

Pachic Fulvudands

Thaptic Fulvudands

Typic Fulvudands

Hydric Fulvudands

Typic Hapludands

Hydric Hapludands

Aquic Hapludands

Oxyaquic Hapludands

Typic Hapludands

Hydric Hapludands

Spodosols

Spodosols

Histic Epiaquods

Histic Endoaquods

Endoaquods

Aquic Haplorthods

Epiaquods

Aquic Haplorthods 
Table 3.1 (continued)

Soil classification system of Japan (2017)

Great group

\begin{tabular}{l|l|l|}
\hline & Group & \\
\cline { 2 - 3 } & & Subgroup \\
\hline & & Haplic \\
\hline
\end{tabular}

E.【Fluvic soils】

\begin{tabular}{l|l|l|}
\hline & Fluvic Paddy soils & \\
\hline & & Albic \\
\hline & & Epi-gleyed \\
\hline & & Endoaeric \\
\hline & & Aquic \\
\hline & & Haplic \\
\hline & Gley Fluvic soils & \\
\hline
\end{tabular}

\begin{tabular}{l|l|l|}
\hline & Thioy Fluvic soils \\
& & \\
\hline
\end{tabular}

\begin{tabular}{l|l|l|}
\hline & & Peaty \\
\hline & & Humic \\
\hline & & Epi-gray \\
\hline & & Strong \\
\hline & & Mottled \\
\hline & Gray Fluvic soils & \\
\hline
\end{tabular}

\begin{tabular}{l|l|l|}
\hline & Thionic \\
\hline & & Peaty \\
\hline
\end{tabular}

\begin{tabular}{l|l|l|}
\hline & Humic \\
\hline
\end{tabular}

\begin{tabular}{l|l|l|}
\hline & & Epi-gleyed \\
\hline & & Gleyic \\
\hline & Thapto-andic \\
\hline & $\begin{array}{l}\text { Brown Fluvic } \\
\text { soils }\end{array}$ & Haplic \\
\hline & Aquic \\
\hline
\end{tabular}

World reference base for soil resources (2015)

\begin{tabular}{|l|l}
\hline Haplic Podzols & $\begin{array}{l}\text { Haplorthods } \\
\text { Haplocryods }\end{array}$
\end{tabular}

Fluvisols, Anthrosols

Hydragric Anthrosols (Fluvic)

Hydragric Anthrosols (Fluvic)

Hydragric Anthrosols (Fluvic)

Hydragric Anthrosols (Fluvic)

Hydragric Anthrosols (Fluvic)

Hydragric Anthrosols (Fluvic)

Fluvic Gleysols

Fluvic Thionic Gleysols

Fluvic Histic Gleysols

Fluvic Umbric Gleysols

Fluvic Gleysols

Fluvic Reductigleyic Gleysols

Fluvic Oxygleyic Gleysols

Gleyic Fluvisols

Gleyic Fluvisols

Gleyic Histic Fluvisols

Gleyic Umbrisols

Gleyic Fluvisols

Gleyic Fluvisols

Gleyic Fluvisols

Gleyic Fluvisols

Fluvisols

Fluvisols (Oxyaquic)
Inceptisols, Entisols

Anthraquic Eutrudepts

Aeric Epiaquepts

Anthraquic Eutrudepts

Aeric Epiaquepts

Typic Epiaquepts

Anthraquic Eutrudepts

Aeric Epiaquepts

Typic Epiaquepts

Anthraquic Eutrudepts

Aeric Epiaquepts

Aquents, Aquepts, Aquepts

Sulfic Endoaquepts

Sulfic Hydraquents

Sulfic Fluvaquents

Thapto-Histic Hydraquents

Thapto-Histic Fluvaquents

Typic Hydraquents

Mollic Fluvaquents

Typic Fluvaquents

Typic Hydraquents

Typic Hydraquents

Typic Fluvaquents

Aquepts, Aquents

Sulfaqepts

Sulfic Endoaquepts

Sulfic Fluvaquents

Fluvaquentic Endoaquepts

Thapto-Histic Fluvaquents

Humaquepts

Mollic Fluvaquents

Fluvaquentic Endoaquepts

Typic Endoaquepts

Typic Fluvaquents

Typic Endoaquepts

Typic Fluvaquents

Aquandic Endoaquepts

Aquandic Fluvaquents

Fluvaquentic Endoaquepts

Typic Fluvaquents

Typic Psammaquents

Udifluvents, Psamments

Oxyaquic Udifluvents

Aquic Udipsamments 
Table 3.1 (continued)

Soil classification system of Japan (2017)

Great group

\begin{tabular}{l|l|l|}
\hline & Group & Subgroup \\
\hline & & Humic \\
\hline & & Protoanthraquic \\
\hline & $\begin{array}{l}\text { Regosolic Fluvic } \\
\text { soils }\end{array}$ & Haplic \\
\hline & Aquic \\
\hline & Haplic \\
\hline
\end{tabular}

F. 【Red-Yellow soils】

Argic

Red-Yellow soils

\begin{tabular}{l|l|l|}
\hline & Anthraquic \\
\hline & Albic
\end{tabular}

\begin{tabular}{|l|l|}
\hline & Albic \\
\end{tabular}

\begin{tabular}{l|l|l|}
\hline & Pseudogleyic \\
\hline & Aquic \\
\hline
\end{tabular}

\begin{tabular}{l|l|l}
\hline & Humic \\
\hline & Reddish \\
\hline
\end{tabular}

\begin{tabular}{l|l|}
\hline & \\
\hline & Cambic
\end{tabular}

Red-Yellow soils

G. 【Stagnic soils】

Stagnogley soils

Anthraquic

Albic

Pseudogleyic
World reference base for soil

UUSDA Soil Taxonomy (2014) resources (2015)

\begin{tabular}{|l|l|}
\hline Gleyic Umbrisols & Mollic Udifluvents \\
\hline Fluvisols (Oxyaquic) & $\begin{array}{l}\text { Oxyaquic Udifluvent } \\
\text { Aquic Udipsamments }\end{array}$
\end{tabular}

\begin{tabular}{|l|l} 
Fluvisols & Typic Udifluvents
\end{tabular}

Typic Udipsamments

\begin{tabular}{l|l} 
Fluvisols & Udifluvents, Psamments
\end{tabular}

\begin{tabular}{l|l} 
Fluvisols (Oxaquic) & Oxyaquic Udifluvents
\end{tabular}

Oxyaquic Udipsamments

\begin{tabular}{l|l} 
Fluvisols & Typic Udifluvents
\end{tabular}

Typic Udipsamments

Alisols, Acrisols, Cambisols $\quad$ Udults, Udepts

Alisols, Acrisols, Lixisols Udults

\begin{tabular}{l|l} 
Alic Stagnosols & Anthraquic Paleudults
\end{tabular}

\begin{tabular}{l|l} 
Acric Stagnosols & Aquic Hapludults
\end{tabular}

Stagnic Albic Alisols $\quad$ Typic Paleudults

\begin{tabular}{l|l} 
Albic Acrisols & Aquic Paleudults
\end{tabular}

Typic Hapludults

Aquic Hapludults

Stagnic Alisols $\quad$ Aquic Paleudults

Stagnic Acrisols $\quad$ Aquic Hapludults

Gleyic Alisols

Gleyic Acrisols

Aquic Paleudults

Aquic Hapludults

Alic Umbrisols $\quad$ Typic Haplohumults

Acric Umbrisols $\quad$ Humic Hapludults

\begin{tabular}{l|l} 
Chromic Alisols & Typic Paleudults
\end{tabular}

Chromic Acrisols $\quad$ Typic Hapludults

Haplic $\quad$ Haplic Alisols

Haplic Acrisols

Typic Paleudults

Typic Hapludults

Cambisols

Udepts

Stagnosols

Gleyic Cambisols

Anthraquic Eutrudepts

Stagnic Cambisols

Aquic Dystrudepts

Typic Dystrudepts

Gleyic Cambisols

Aquic Dystrudepts

Oxyaquic Dystrudepts

Aquic Dystrudepts

Oxyaquic Dystrudepts

\begin{tabular}{l|l} 
Cambic Umbrisols & Humic Dystrudepts
\end{tabular}

\begin{tabular}{l|l} 
Chromic Cambisols & Typic Dystrudepts
\end{tabular}

\begin{tabular}{l|l} 
Reddish & Chstric Cambisols \\
\hline Andic & Dystic
\end{tabular}

Andic Dystrudepts

Dystric Cambisols

Oxyaquic Dystrudepts

Typic Dystrudepts

Gleysols, Stagnosols, Anthrosols Aquepts, Aquults, Aquents

Gleysols, Anthrosols
Epiaquepts, Endoaquepts, Endoaquents 
Table 3.1 (continued)

\begin{tabular}{|c|c|c|c|c|}
\hline \multicolumn{3}{|c|}{ Soil classification system of Japan (2017) } & \multirow{4}{*}{$\begin{array}{l}\text { World reference base for soil } \\
\text { resources (2015) }\end{array}$} & \multirow[t]{4}{*}{ UUSDA Soil Taxonomy (2014) } \\
\hline \multirow{2}{*}{\multicolumn{3}{|c|}{ Great group }} & & \\
\hline & Group & & & \\
\hline & & Subgroup & & \\
\hline & & Anthraquic & $\begin{array}{l}\text { Anthraquic Gleysols } \\
\text { Hydroargic Anthrosols }\end{array}$ & Typic Epiaquepts \\
\hline & & Epi-peaty & Histic Gleysols & $\begin{array}{l}\text { Typic Epiaquepts } \\
\text { Typic Endoaquents }\end{array}$ \\
\hline & & Humic & Umbric Gleysols & $\begin{array}{l}\text { Typic Humaquepts } \\
\text { Humaquptic Endoaquents }\end{array}$ \\
\hline & & Haplic & Haplic Gleysols & $\begin{array}{l}\text { Typic Epiaquepts } \\
\text { Typic Endoaquents }\end{array}$ \\
\hline & Pseudogley soils & & Stagnosols, Gleysols & Aquepts, Aquults, Aquents \\
\hline & & Anthraquic & Anthraquic Stagnosols & $\begin{array}{l}\text { Typic Epiaquepts } \\
\text { Typic Epiaquults }\end{array}$ \\
\hline & & Groundwater-aquic & $\begin{array}{l}\text { Endogleyic Stagnosols } \\
\text { Anthraquic Gleysols } \\
\text { Haplic Gleysols }\end{array}$ & $\begin{array}{l}\text { Typic Endoaquepts } \\
\text { Typic Endoaquents }\end{array}$ \\
\hline & & Humic & Umbric Stagnosols & $\begin{array}{l}\text { Typic Humaquepts } \\
\text { Typic Umbraquults }\end{array}$ \\
\hline & & Aeric & Haplic Stagnosols & $\begin{array}{l}\text { Aeric Epiaquepts } \\
\text { Aeric Epiaquults }\end{array}$ \\
\hline & & Haplic & Haplic Stagnosols & $\begin{array}{l}\text { Typic Epiaquepts } \\
\text { Typic Epiaquults }\end{array}$ \\
\hline \multicolumn{2}{|l|}{ H.【Eutrosols】 } & & Luvisols, Cambisols & Udalfs, Udepts \\
\hline & $\begin{array}{l}\text { Magnesian } \\
\text { Eutrosols }\end{array}$ & & Luvisols, Cambisols & Udalfs, Udepts \\
\hline & & Argic & $\begin{array}{l}\text { Leptic Luvisols (Chromic) } \\
\text { Leptic Luvisols (Rhodic) } \\
\text { Haplic Luvisols (Chromic) } \\
\text { Haplic Luvisols (Rhodic) }\end{array}$ & $\begin{array}{l}\text { Typic Paleudalfs } \\
\text { Typic Rhodualfs } \\
\text { Typic Hapludalfs }\end{array}$ \\
\hline & & Haplic & $\begin{array}{l}\text { Leptic Cambisols (Eutric) } \\
\text { Haplic Cambisols (Eutric) }\end{array}$ & $\begin{array}{l}\text { Lithic Eutrudepts } \\
\text { Typic Eutrudepts }\end{array}$ \\
\hline & $\begin{array}{l}\text { Calcareous } \\
\text { Eutrosols }\end{array}$ & & Luvisols, Cambisols & Udalfs, Udepts \\
\hline & & Argic & $\begin{array}{l}\text { Leptic Luvisols } \\
\text { Haplic Luvisols }\end{array}$ & $\begin{array}{l}\text { Typic Paleudalfs } \\
\text { Typic Rhodualfs } \\
\text { Typic Hapludalfs }\end{array}$ \\
\hline & & Haplic & $\begin{array}{l}\text { Leptic Cambisols (Eutric) } \\
\text { Haplic Cambisols (Eutric) }\end{array}$ & $\begin{array}{l}\text { Lithic Eutrudepts } \\
\text { Typic Eutrudepts }\end{array}$ \\
\hline \multirow{6}{*}{$\begin{array}{l}\text { I. 【Brown Forest } \\
\text { soils】 }\end{array}$} & & & Camibisols, Stagnosols & Udepts \\
\hline & $\begin{array}{l}\text { Brown Forest } \\
\text { soils }\end{array}$ & & Camibisols, Stagnosols & Udepts \\
\hline & & Anthraquic & Anthraquic Stagnosols & $\begin{array}{l}\text { Anthraquic Eutrudepts } \\
\text { Aquic Dystrudepts }\end{array}$ \\
\hline & & Andic & Dystric Cambisols & $\begin{array}{l}\text { Andic Dystrudepts } \\
\text { Andic Eutrudepts } \\
\text { Lithic Dystrudepts }\end{array}$ \\
\hline & & Podzolic & Dystric Cambisols & $\begin{array}{l}\text { Typic Dystrudepts } \\
\text { Lithic Dystrudepts }\end{array}$ \\
\hline & & Humic & Cambic Umbrisols & Humic Dystrudepts \\
\hline
\end{tabular}


Table 3.1 (continued)

\begin{tabular}{|c|c|c|c|c|}
\hline \multicolumn{3}{|c|}{ Soil classification system of Japan (2017) } & \multirow{4}{*}{$\begin{array}{l}\text { World reference base for soil } \\
\text { resources (2015) }\end{array}$} & \multirow[t]{4}{*}{ UUSDA Soil Taxonomy (2014) } \\
\hline \multicolumn{3}{|l|}{ Great group } & & \\
\hline & Group & & & \\
\hline & & Subgroup & & \\
\hline & & & Dystric Cambisols (Humic) & \\
\hline & & Thapto-red-yellow & $\begin{array}{l}\text { Dystric Cambisols } \\
\text { Haplic Alisols }\end{array}$ & $\begin{array}{l}\text { Typic Dystrudepts } \\
\text { Typic Paleudults } \\
\text { Inceptic Hapludults } \\
\text { Typic Hapludults }\end{array}$ \\
\hline & & Aquic & Gleyic Cambisols (Oxaquic) & $\begin{array}{l}\text { Aquic Dystrudepts } \\
\text { Oxyaquic Dystrudepts } \\
\text { Lithic Dystrudepts }\end{array}$ \\
\hline & & Epi-gleyed & Gleyic Cambisols & $\begin{array}{l}\text { Aquic Dystrudepts } \\
\text { Oxyaquic Dystrudepts } \\
\text { Lithic Dystrudepts }\end{array}$ \\
\hline & & Eutric & Eutric Cambisols & $\begin{array}{l}\text { Lithic Eutrudepts } \\
\text { Typic Eutrudepts }\end{array}$ \\
\hline & & Haplic & Dystric Cambisols & $\begin{array}{l}\text { Typic Dystrudepts } \\
\text { Lithic Dystrudepts }\end{array}$ \\
\hline \multirow[t]{18}{*}{ J.【Regosols】 } & & & $\begin{array}{l}\text { Regosols, Arenosols, Leptosols, } \\
\text { Phaeozems }\end{array}$ & Entisols, Mollisols \\
\hline & $\begin{array}{l}\text { Volcanogeous } \\
\text { Regosols }\end{array}$ & & Tephric Regosols & Orthents \\
\hline & & Aquic & Gleyic Tephric Regosols & Aquic Udorthents \\
\hline & & Haplic & Tephric Regosols & Vitrandic Udorthents \\
\hline & Sandy Regosols & & Arenosols & Udipsamments \\
\hline & & Calcaric & Calcaric Arenosols & Typic Udipsamments \\
\hline & & Aquic & Gleyic Arenosols & $\begin{array}{l}\text { Aquic Udipsamments } \\
\text { Oxyaquic Udipsamments }\end{array}$ \\
\hline & & Haplic & Arenosols & Typic Udipsamments \\
\hline & Lithosols & & Leptosols & Udorthents, Rendolls \\
\hline & & Calcaric & $\begin{array}{l}\text { Calcaric Rendzic Leptosols } \\
\text { Calcaric Leptosols }\end{array}$ & $\begin{array}{l}\text { Lithic Haprendolls } \\
\text { Lithic Udorthents }\end{array}$ \\
\hline & & Aquic & Leptosols (Gleyic) & Lithic Udorthents \\
\hline & & Haplic & Leptosols & Lithic Udorthents \\
\hline & $\begin{array}{l}\text { Terrestrial } \\
\text { Regosols }\end{array}$ & & Regosols & Udorthents \\
\hline & & Marlitic & $\begin{array}{l}\text { Calcaric Regosols } \\
\text { Calcaric Leptosols }\end{array}$ & $\begin{array}{l}\text { Typic Udorthents } \\
\text { Lithic Udorthents }\end{array}$ \\
\hline & & Calcaric & $\begin{array}{l}\text { Calcaric Regosols } \\
\text { Calcaric Phaeozems }\end{array}$ & $\begin{array}{l}\text { Typic Haprendolls } \\
\text { Typic Udorthents }\end{array}$ \\
\hline & & Para-lithic & $\begin{array}{l}\text { Dystric Regosols } \\
\text { Dystric Leptosols }\end{array}$ & $\begin{array}{l}\text { Typic Udorthents } \\
\text { Lithic Udorthents }\end{array}$ \\
\hline & & Granitic & $\begin{array}{l}\text { Skeletic Regosols } \\
\text { Skeletic Leptosols }\end{array}$ & $\begin{array}{l}\text { Typic Udorthents } \\
\text { Lithic Udorthents }\end{array}$ \\
\hline & & Haplic & Dystric Regosols & Typic Udorthents \\
\hline
\end{tabular}

Please note that it shows only representative classification names corresponding to each classification and does not cover all cases 